TMUP-HEL-9503

March 29, 1995

Revised: June 16, 1995

hep-ph/9504222

\title{
Neutrino Flavor Mixing Constrained by Accelerator and Reactor Experiments and Solar Neutrino Observation*
}

\author{
Hisakazu Minakata ${ }^{\dagger}$ \\ Department of Physics, Tokyo Metropolitan University, \\ 1-1 Minami-Osawa, Hachioji, Tokyo 192-03 Japan
}

\begin{abstract}
We derive the constraints imposed on neutrino masses and mixing angles by performing a combined analysis of the data from the Los Alamos and the other terrestrial neutrino oscillation experiments with the assumption of the flavor-mixing solutions to the solar neutrino problem. In a three-flavor mixing scheme which ignores the possibility of sterile neutrinos, we obtain severe constraints on the pattern of masses and flavor mixing of neutrinos. For example, we show that in the standard Kobayashi-Maskawa type mixing matrix $s_{13}^{2} \lesssim 10^{-2}$ independent of the choice of the solar neutrino solutions. The constraint from the double $\beta$ decay is also discussed.
\end{abstract}

\footnotetext{
${ }^{*}$ Work supported in part by Grant-in-Aid for Scientific Research of the Ministry of Education, Science and Culture \#0560355.

†email: minakata@phys.metro-u.ac.jp
} 
Recently the Liquid Scintillator Neutrino Detector(LSND) group in Los Alamos has reported positive evidence for the neutrino oscillation events $\overline{\nu_{\mu}} \rightarrow \overline{\nu_{e}}$ [1, 2]. If confirmed by continuing runs, it provides the first direct evidence for nonvanishing masses and flavor mixings of neutrinos. It is certainly an urgent problem to illuminate all possible consequences of this discovery [3].

Before hearing this exciting report we have been speculating about the neutrino masses and mixing with the help of information of astrophysical neutrinos detected by the various underground detectors. Among them the most popular one is the solar neutrino deficit which lasts more than 20 years $\llbracket$, but still refuses resolution by the standard solar model [5]. Now the solar neutrino deficit is confirmed to exist by the four different experiments, the chlorine [6], the Kamiokande [7] and the two gallium experiments [8,9]. A less popular but an equally important piece of information comes from the atmospheric neutrino anomaly, a deficit in the ratio $\left(\nu_{\mu}+\overline{\nu_{\mu}}\right) /\left(\nu_{e}+\overline{\nu_{e}}\right)$, which was first claimed to exist by the Kamiokande group [10], and subsequently supported by the IMB [11] and the Soudan 2 [12] experiments. The new "multi-GeV" data sample reported by the Kamiokande group [13] provides strong support for the interpretation of the atmospheric neutrino anomaly in terms of the neutrino oscillation.

In this paper we discuss possible implications of the LSND result by performing a combined analysis of the data with that of solar neutrino observation and the reactor experiments. Our analysis is based on the three-flavor mixing scheme of neutrinos without introducing any sterile species. We will recognize, as the analysis proceeds, that it is crucial to include the information of the disappearance experiments. The most stringent limits achieved for $\nu_{e}$ and $\nu_{\mu}$ channels are the ones reported by the Bugey [14] and the CDHS 15 groups, respectively.

The most disturbing feature of this restricted framework is, of course, that it cannot accomodate all of them, the LSND result, the solar neutrino deficit, and the atmospheric neutrino anomaly. The reason is very simple; three mass scales (more precisely, mass-squared differences) which are involved in these three phenomena are too far apart from each other 
to be accomodated in the three-generation scheme [3]. In the present paper we therefore omit atmospheric neutrino data from the analysis. A combined analysis of the alternative combination, LSND-Atmospheric neutrino, is presented elsewhere [16].

To orient our analysis and to focus some important points we summarize below the features of the LSND and the disappearance experiments, and the flavor mixing solution to the solar neutrino problem.

(1) The LSND experiment [2,28,29]:

By using low energy $\overline{\nu_{\mu}}$ beam from stopped muons the LSND group is able to perform the appearance experiment $\overline{\nu_{\mu}} \rightarrow \overline{\nu_{e}}$ which can probe the neutrino oscillation parameters up to $\Delta m^{2}=0.2 \mathrm{eV}^{2}\left(0.5 \mathrm{eV}^{2}\right)$ for $\sin ^{2} 2 \theta=0.01(0.001)$. Such great sensitivity has been achieved by the intense pion beam from the LAMPF accelerator and by the low enough energies of $\overline{\nu_{\mu}}$ beam. After oscillating into $\overline{\nu_{e}}$, it produces positrons via the reaction $\overline{\nu_{e}} p \rightarrow e^{+} n$ which are measured in the energy window $36 \mathrm{MeV}<\mathrm{E}_{e}<60 \mathrm{MeV}$. The produced neutrons are captured by protons through the reaction $\mathrm{np} \rightarrow \mathrm{d} \gamma$, whose gamma rays of energy $2.2 \mathrm{MeV}$ can serve for delayed coincidence. The experimental group may have observed excess in gamma ray-correlated events over the estimated background.

(2) The disappearance experiments [14.15]:

This type of experiment measures the attenuation of neutrino beam from a reactor or an accelerator. The intensity of $\overline{\nu_{e}}$ beam from the reactor at Bugey is measured by the ${ }^{6}$ Li-loaded liquid scintillator located at 15, 40 and 95m from the reactor [14]. From the viewpoint of neutrino mixing the experiment measures $1-P\left(\overline{\nu_{e}} \rightarrow \overline{\nu_{e}}\right)$, where $P\left(\overline{\nu_{e}} \rightarrow \overline{\nu_{e}}\right)$ implies the survival probability of electron antineutrinos. The resulting bound is rather severe and may be summarized as $1-P\left(\overline{\nu_{e}} \rightarrow \overline{\nu_{e}}\right) \leq 5 \%$ including the statistical and the systematic uncertainties. A comparable limit is achieved for $\nu_{\mu}$ channel by the CDHS group [15] using $\nu_{\mu}$ beam from the CERN PS.

(3) The flavor-mixing solutions to the solar neutrino problem:

The most popular and perhaps most appealing solution to the solar neutrino problem is the one provided by the Mikhyev-Smirnov-Wolfenstein(MSW) mechanism [17]. It 
utilizes the (by now familiar) mechanism of resonant enhancement of neutrino flavor conversion in solar matter. At the moment there exist two options in this type of solution; the small-angle (nonadiabatic), and the large-angle solutions. The characteristic values of the neutrino mixing parameters are determined by, for example, an extensive analysis by Hata and Langacker [18] and are given as $\Delta m^{2} \simeq 6 \times 10^{-6} \mathrm{eV}^{2}, \quad \sin ^{2} 2 \theta \simeq 7 \times 10^{-3}$, and $\Delta m^{2} \simeq 9 \times 10^{-6} \mathrm{eV}^{2}, \quad \sin ^{2} 2 \theta \simeq 0.6$, for the small-angle and the large-angle solutions, respectively. Their analysis is done with the two-flavor mixing scheme, and $\Delta m^{2}$ and $\theta$ indicate the mass-squared difference and the mixing angle, respectively. We should mention that there still exists the possibility of vacuum neutrino oscillation as a mechanism for the solar neutrino deficit. This is the old solution [4] but one still alive [19].

One of the most important features in our combined analysis of the LSND and the solar neutrino solution is that a huge mass hierarchy is involved. (In fact, it is not really the hierarchy in mass but is the hierarchy in the mass-difference.) One may classify the hierarchy of neutrino masses into two types:

$$
\begin{array}{ll}
\text { (a) } m_{1}^{2} \approx m_{2}^{2} \gg m_{3}^{2} & \text { (b) } m_{3}^{2} \gg m_{2}^{2} \approx m_{1}^{2}
\end{array}
$$

Here the symbols $\approx$ and $\gg$ imply the difference by $\lesssim 10^{-5} \mathrm{eV}^{2}$ and $\sim 1-10 \mathrm{eV}^{2}$, respectively. In (11) we have chosen the third state as the grossly departed mass eigenstate. The other types of mass hierarchies which can be obtained by permuting 1, 2, and 3 can be taken care of by an appropriate choice of angles because they merely represent relabeling of the mass eigenstates. Unlike the case of vacuum neutrino oscillation [16 the relative magnitude of the masses connected by $\approx$ does have important meaning and will be discussed below.

We briefly review the neutrino oscillation with three flavors of neutrinos. We define the neutrino mixing matrix $U$ which relates the flavor eigenstate $\nu_{\alpha}(\alpha=e, \mu, \tau)$ and the masseigenstate $\nu_{i}(i=1,2,3)$ in vacuum as $\nu_{\alpha}=U_{\alpha i} \nu_{i}$. In this paper we assume CP invariance. The evolution equation of the flavor eigenstate takes the form

$$
i \frac{d}{d x} \nu_{\alpha}=\frac{m_{i}^{2}}{2 E} \delta_{i j} U_{\alpha i}\left(U^{-1}\right)_{j \beta} \nu_{\beta}+\delta_{\alpha e} \delta_{e \beta} a_{e}(x) \nu_{\beta},
$$


where $a_{e}(x)=\sqrt{2} G_{F} N_{e}(x)$ indicates the effect of matter potential which affects only electron neutrinos. Here, $G_{F}, E$ and $N_{e}$ denote, in order, the Fermi constant, the neutrino energy, and the electron number density in the sun.

Without matter effect the equation (2) can be easily integrated to yield the oscillation probability $\nu_{\alpha} \rightarrow \nu_{\beta}$ as

$$
\begin{aligned}
P\left(\nu_{\beta} \rightarrow \nu_{\alpha}\right) & =P\left(\overline{\nu_{\beta}} \rightarrow \nu_{-}^{-} \alpha\right) \\
& =\delta_{\alpha \beta}-4 \sum_{j>i} U_{\alpha i} U_{\beta i} U_{\alpha j} U_{\beta j} \sin ^{2}\left(\frac{\Delta m_{i j}^{2} L}{4 E}\right) .
\end{aligned}
$$

The mass-squared difference $\Delta m_{i j}^{2}$ is defined as $\Delta m_{i j}^{2} \equiv m_{j}^{2}-m_{i}^{2}(j>i)$ in this paper. Notice that when we discuss the neutrino evolution in matter the sign of $\Delta m_{i j}^{2}$ does have physical meaning, unlike the case of vacuum neutrino oscillation (3).

With matter effect the equation becomes complicated but it is tractable because of the mass hierarchy involved in our analysis. To make our discussion transparent we specify the form of the mixing matrix in a form $U=U_{23} U_{13} U_{12}$,

$$
U=\left[\begin{array}{ccc}
1 & 0 & 0 \\
0 & c_{23} & s_{23} \\
0 & -s_{23} & c_{23}
\end{array}\right]\left[\begin{array}{ccc}
c_{13} & 0 & s_{13} \\
0 & 1 & 0 \\
-s_{13} & 0 & c_{13}
\end{array}\right]\left[\begin{array}{ccc}
c_{12} & s_{12} & 0 \\
-s_{12} & c_{12} & 0 \\
0 & 0 & 1
\end{array}\right]
$$

where $U_{23}, U_{13}$, and $U_{12}$ denote the three matrices in (田), in order, from left to right. Here $c_{i j}$ and $s_{i j}$ are the short-hand notations for $\cos \theta_{i j}$ and $\sin \theta_{i j}$, respectively. This is nothing but the standard form of the Kobayashi-Maskawa matrix [20], which is now employed as the neutrino mixing matrix. We note that $\mathrm{CP}$ invariance renders the three angles real and they can all be made to lie in the first quadrant by an appropriate redefinition of neutrino phases.

The definition of the mixing matrix (4) is convenient in dealing with the mass patterns (1) in which 1-2 level crossing is responsible for the solar neutrino deficit. If one wants to discuss the other type of mass pattern which can be obtained by permuting 1,2 , and 3 one may make a different choice of the $U$ matrix (i.e., redefinition of angles) convenient 
for them. For example, $U=U_{13} U_{12} U_{23}$ for 2-3 level crossing. We note that one can take $\theta_{12} \leq \frac{\pi}{4}$ so that $\nu_{e}$ is close to $\nu_{1}$ under the two-level crossing approximation. It does not hurt the generality of our analysis because the constraints from the terrestrial experiments to be discussed below do not involve $\theta_{12}$.

We examine the MSW effect in the three-flavor mixing scheme. We multiply, following Kuo and Pantaleone [21], $U_{13}^{-1} U_{23}^{-1}$ to the equation (21) to obtain the evolution equation for the modified neutrino basis $\tilde{\nu_{\beta}}=\left(U_{13}^{-1} U_{23}^{-1}\right)_{\beta \alpha} \nu_{\alpha}$ :

$$
i \frac{d}{d x}\left[\begin{array}{c}
\tilde{\nu_{e}} \\
\tilde{\nu_{\mu}} \\
\tilde{\nu_{\tau}}
\end{array}\right]=\left[\begin{array}{ccc}
-\Delta \cos 2 \theta_{12}+c_{13}^{2} a_{e} & \Delta \sin 2 \theta_{12} & \frac{1}{2} \sin 2 \theta_{13} a_{e} \\
\Delta \sin 2 \theta_{12} & \Delta \cos 2 \theta_{12} & 0 \\
\frac{1}{2} \sin 2 \theta_{13} a_{e} & 0 & \sum+s_{13}^{2} a_{e}
\end{array}\right]\left[\begin{array}{c}
\tilde{\nu_{e}} \\
\tilde{\nu_{\mu}} \\
\tilde{\nu_{\tau}}
\end{array}\right],
$$

where we have used simplified notation $\Delta \equiv \frac{\Delta m_{12}^{2}}{4 E}$ and $\sum \equiv \frac{1}{2}\left(\Delta m_{13}^{2}+\Delta m_{23}^{2}\right)$. Note that the sign of $\Delta$ has physical significance.

From (5) one realizes that the effective two-level crossing approximation is justified unless $\sin 2 \theta_{13} a_{e}$ is extraordinarily large compared with other elements, which is not the case in our problem. Moreover, an evaluation of the perturbative corrections to the energy eigenvalues due to this off-diagonal term reveals that they are of the order of $-\left(\sin 2 \theta_{13} a_{e}\right)^{2} /\left|\sum\right|[21]$ which is negligible for the mass hierarchy $\Delta m_{13}^{2} \approx \Delta m_{23}^{2} \gg \Delta m_{12}^{2}$. Notice that this is true for both of the types-a and -b mass patterns given in (11). Also it can be shown that the correction to the difference between two energy eigenvalues in matter vanishes at the resonance point. Therefore, the off-diagonal terms affect the discussion of the adiabaticity condition in the effective two-level problem only through higher-order corrections.

Having established the validity of the effective two-level approximation we proceed to the combined analysis. We first derive the approximate formulas for the terrestrial neutrino experiments, taking into account the mass hierarchy and the experimental parameters. With mass hierarchy (1) the oscillation probability consists of two terms, one involving large $\Delta m_{13}^{2}$ and other small $\Delta m_{12}^{2}$. The coefficient of the former term have a simplified expression due to the orthogonality of the mixing matrix [16]. The latter term is smaller by factors of 
$\left(\Delta m_{12}^{2} / \Delta m_{13}^{2}\right)^{2} \leq 10^{-10}$ compared with the former. So it can be safely ignored.

In certain cases, the argument of the sine function with larger mass difference takes the large values, e. g., 10-100 for $\Delta m_{13}^{2}=1-10 \mathrm{eV}^{2}$ with $\mathrm{E}=4 \mathrm{MeV}$ and $\mathrm{L}=40 \mathrm{~m}$, the typical parameters in the Bugey experiment. Therefore, it can be averaged to be $\frac{1}{2}$ and we obtain, as the formula for the Bugey disappearance experiment,

$$
1-P\left(\overline{\nu_{e}} \rightarrow \overline{\nu_{e}}\right)=2 c_{13}^{2} s_{13}^{2} .
$$

For the LSND experiments the arguments of sine factors with $\Delta m_{13}^{2}$ and $\Delta m_{23}^{2}$ are of order unity and so we cannot average. The oscillation probability in the LSND experiment is thus

$$
P\left(\overline{\nu_{\mu}} \rightarrow \overline{\nu_{e}}\right)=4 s_{23}^{2} c_{13}^{2} s_{13}^{2} \sin ^{2}\left(\frac{\Delta m_{13}^{2} L}{4 E}\right) .
$$

Similarly, the oscillation probabilities of $\nu_{\mu} \rightarrow \nu_{\mu}$ and $\nu_{\mu} \rightarrow \nu_{\tau}$ channels take the forms

$$
\begin{gathered}
1-P\left(\nu_{\mu} \rightarrow \nu_{\mu}\right)=4 s_{23}^{2} c_{13}^{2}\left(1-s_{23}^{2} c_{13}^{2}\right) \sin ^{2}\left(\frac{\Delta m_{13}^{2} L}{4 E}\right), \\
P\left(\nu_{\mu} \rightarrow \nu_{\tau}\right)=4 c_{23}^{2} s_{23}^{2} c_{13}^{4} \sin ^{2}\left(\frac{\Delta m_{13}^{2} L}{4 E}\right) .
\end{gathered}
$$

From the data of the Bugey and the LSND experiments described earlier we require

$$
\begin{array}{r}
c_{13}^{2} s_{13}^{2} \leq 2.5 \times 10^{-2} \equiv \delta \\
s_{23}^{2} c_{13}^{2} s_{13}^{2} \equiv \epsilon \lesssim 10^{-3}
\end{array}
$$

where we have defined $\delta$ and $\epsilon$ such that $2 \delta$ and $4 \epsilon$ imply the attenuation of $\overline{\nu_{e}}$ in the Bugey and the "rate" in the LSND experiments, respectively. Since the latter quantity is still subject to the uncertainty [28,29], we take a conservative attitude and assume that $\epsilon$ is less than or equal to $\sim 10^{-3}$. From $(\sqrt{10})$ and (11) it follows that $\theta_{13}$ must be either small or close to $\frac{\pi}{2}$

$$
\epsilon \leq\left(\begin{array}{c}
s_{13}^{2} \\
c_{13}^{2}
\end{array}\right) \leq 2.6 \times 10^{-2} \simeq \delta
$$


The angle $s_{23}$ is severely constrained by the the $\nu_{\mu}$ disappearance experiment. The CDHS group [15] obtained the constraint on $\sin ^{2} 2 \theta$ (in two-flavor scheme), $\sin ^{2} 2 \theta \lesssim 0.1$ in the mass range $1 \mathrm{eV}^{2} \leq \Delta m_{13}^{2} \leq 10 \mathrm{eV}$, where the constraint from the CDHS experiment is most stringent. Using this with (8) we obtain the bound on $s_{23}^{2}$ as

$$
\left(\begin{array}{c}
s_{23}^{2} c_{13}^{2} \\
1-s_{23}^{2} c_{13}^{2}
\end{array}\right) \lesssim 2.5 \times 10^{-2} \simeq \delta
$$

In large- $\Delta m_{13}^{2}$ region a better bound can be obtained from $\nu_{\mu} \rightarrow \nu_{\tau}$ oscillation experiment. Using the data of Fermilab E531 experiment [22] with (9) we obtain an approximate expression of the $\Delta m^{2}$-dependent bound in the same mass range:

$$
c_{23}^{2} s_{23}^{2} c_{13}^{4} \lesssim 0.25 \times\left(\frac{\Delta m_{13}^{2}}{1 \mathrm{eV}^{2}}\right)^{-2}
$$

We will show below that the solar neutrino solutions force us to choose the small- $s_{13}$ solution out of (12). Using this information apriori with (13) and (14) we obtain the bound on $s_{23}^{2}$,

$$
\left(\begin{array}{c}
s_{23}^{2} \\
c_{23}^{2}
\end{array}\right) \lesssim 2.5 \times 10^{-2} \times \begin{cases}1 & \left(1 \mathrm{eV}^{2} \leq \Delta \mathrm{m}_{13}^{2} \leq 3.3 \mathrm{eV}^{2}\right) \\
\left(\frac{\Delta m_{13}^{2}}{3.3 \mathrm{eV}^{2}}\right)^{-2} & \left(3.3 \mathrm{eV}^{2} \leq \Delta \mathrm{m}_{13}^{2} \leq 10 \mathrm{eV}^{2}\right)\end{cases}
$$

Now let us turn to the constraint implied by the solar neutrino solutions. Using the local two-level crossing approximation established above $\Delta m_{12}^{2}$ and $s_{12}^{2}$ are determined to be

$$
\Delta m_{12}^{2} \simeq 6 \times 10^{-6} \mathrm{eV}^{2}, \quad s_{12}^{2} \simeq 1.75 \times 10^{-3}, \quad \text { (small-angle) }
$$

and

$$
\Delta m_{12}^{2} \simeq 9 \times 10^{-6} \mathrm{eV}^{2}, \quad s_{12}^{2} \simeq 0.184, \quad \text { (large-angle) }
$$

for the small-angle and the large-angle MSW solutions, respectively. Of course, the masssquared difference $\Delta m_{12}^{2}$ must be positive in order that the MSW mechanism acts for neutrinos, not for antineutrinos. This implies $m_{2}^{2}>m_{1}^{2}$ between almost degenerate neutrino states in (11). 
So far we have discussed the constraints from the terrestrial experiments and that from the MSW solar neutrino solutions separately (except what we did for $s_{23}$ ). While we have obtained highly nontrivial constraints (12)-(14), it would be more interesting if there arise further restrictions by considering these three experimental requirements simultaneously. We show that this in fact occurs.

We demonstrate that the large- $s_{13}$ solution (i. e., $c_{13}^{2} \leq 2.6 \times 10^{-2}$ ) is not acceptable as a solution to the solar neutrino problem. To show this we note the relationship between the flavor basis $\nu_{\alpha}$ and the basis $\tilde{\nu_{\alpha}}$ introduced as a convenient basis for describing the effectively local two-level resonance in neutrino evolution in matter. It is

$$
\left[\begin{array}{c}
\tilde{\nu_{e}} \\
\tilde{\nu_{\mu}} \\
\tilde{\nu_{\tau}}
\end{array}\right]=\left[\begin{array}{ccc}
c_{13} & -s_{23} s_{13} & -c_{23} s_{13} \\
0 & c_{23} & -s_{23} \\
s_{13} & s_{23} c_{13} & c_{23} c_{13}
\end{array}\right]\left[\begin{array}{c}
\nu_{e} \\
\nu_{\mu} \\
\nu_{\tau}
\end{array}\right] \text {. }
$$

It we take the large- $s_{13}$ solution the initial condition $\left[\begin{array}{lll}\nu_{e} & \nu_{\mu} & \nu_{\tau}\end{array}\right] \simeq[1,0,0]$ at the solar core is translated into the initial condition $\left[\begin{array}{ccc}\tilde{\nu_{e}} & \tilde{\nu_{\mu}} & \tilde{\nu_{\tau}}\end{array}\right]=[\sqrt{\delta}, 0,1]$. Since $\tilde{\nu_{\tau}}$ effectively decouples with $2 \times 2$ submatrix which has resonance, as can be seen in (5), it experiences no significant change in the solar interior. Therefore, $\nu_{e}$ 's which departed the solar core just leave the sun with no appreciable attenuation in their flux.' So the large- $s_{13}$ solution cannot explain the solar neutrino deficit. This conclusion is valid for both of the small-angle and the large-angle MSW solutions, because the relation (18) is independent of $s_{12}$. Thus, we are left with the small- $s_{13}$ solution with the additional constraints (15) together with either (16), or (17), depending upon the small-angle and the large-angle MSW solutions, respectively.

\footnotetext{
${ }^{1}$ One can reach the same conclusion by using the local two-level crossing representation of 1-3 channel [21]. In this case " $\tilde{\nu_{\tau}}$ " does not experience a level crossing not because it decouples but because the resonance condition cannot be met due to the mass hierarchy $\Delta m_{12}^{2} \ll \Delta m_{13}^{2} \approx \Delta m_{23}^{2}$ with which we are working.
} 
Now we turn to the discussion of the solar neutrino solution based on the vacuum neutrino oscillations. Because of the mass hierarchy involved, $1-10 \mathrm{eV}^{2} \simeq \Delta m_{13}^{2} \approx \Delta m_{23}^{2} \gg \Delta m_{12}^{2} \simeq$ $10^{-10} \mathrm{eV}^{2}$, the relevant oscillation probability can be written as

$$
P\left(\nu_{e} \rightarrow \nu_{e}\right)=1-2 c_{13}^{2} s_{13}^{2}-4 c_{12}^{2} s_{12}^{2} c_{13}^{4} \sin ^{2}\left(\frac{\Delta m_{12}^{2} L}{4 E}\right)
$$

where the sine-squared terms with $\Delta m_{13}^{2}$ and $\Delta m_{23}^{2}$ are averaged. It is an excellent approximation because the argument of sine takes a very large value, $\simeq 2 \times$ $10^{10}\left(\Delta m^{2} / 1 \mathrm{eV}^{2}\right)(L / 1 \mathrm{AU})(E / 10 \mathrm{MeV})^{-1}$. In view of (19) and noticing the Bugey constraint (10) we realize that the only possible way of having solar neutrino deficit of $\sim 50 \%$ level is to have an effective two-flavor description of $P\left(\nu_{e} \rightarrow \nu_{e}\right)$. Namely, we have to demand

$$
\begin{gathered}
c_{12}^{2} s_{12}^{2} \simeq \frac{1}{4}(=\text { maximum value }) \\
c_{13}^{2} \simeq 1
\end{gathered}
$$

to have a gross deficit. Under the condition (20) the vacuum neutrino oscillation (19) will provide an acceptable solution to the solar neutrino problem (with possible slight changes in mixing parameters) as shown in [23]. Thus, the combined analysis of the LSND and the Bugey experiments with the vacuum mixing solution of the solar neutrino problem again prefers the small- $s_{13}$ solution.

An additional constraint arises in the case of Majorana neutrinos. The quantity

$$
\begin{aligned}
<m_{\nu e}> & =\sum_{j=1}^{3} \eta_{j}\left|U_{e j}\right|^{2} m_{j} \\
& =\eta_{1} c_{12}^{2} c_{13}^{2} m_{1}+\eta_{2} s_{12}^{2} c_{13}^{2} m_{2}+\eta_{3} s_{13}^{2} m_{3}
\end{aligned}
$$

is constrained to be less than $\sim 1 \mathrm{eV}$ by the non-observation of the neutrinoless double $\beta$ decay in various experiments [24]. Notice that we are working with the representation in which the mixing matrix is real under the assumption of CP invariance, and $\eta_{j}= \pm 1$ in (21) is the CP phase.

The constraint from the double $\beta$ decay acts differently for the type-a and the type-b mass hierarchies in (11). In the type-a case there is a chance for cancellation between nearly 
degenerate two masses, but there is no chance in the type-b case because the heavy mass is carried by a unique mass eigenstate.

We, however, encounter a new situation in the consistent solutions obtained in our combined analysis. For the type-b mass hierarchy the double $\beta$ decay constraint is automatically satisfied by the small- $s_{13}^{2}$. On the other hand, the constraint for the type-a mass hierarchy has nontrivial consequences. In the case of small-angle MSW solution, the cancellation between 1 and 2 mass eigenstates is hopeless because $s_{12}^{2}$ is too tiny, $\simeq 10^{-3}$. In the case of large-angle MSW solution the situation is better but we still obtain $<m_{\nu e}>\simeq 1.7 \mathrm{eV}$ for $m_{1}=m_{2}=2.4 \mathrm{eV}$, which is larger by factor of $\sim 2$ than the experimental bound [24]. In the case of vacuum oscillation solution, there exists better chance for cancellation because $s_{12}^{2}$ is large. The double $\beta$ constraint can be cleared with a mild condition $33^{\circ} \leq \theta_{12} \leq 57^{\circ}$. Thus, the double $\beta$ constraint prefers the vacuum oscillation solution for the type-a mass hierarchy, while it is automatically satisfied for the type-b mass pattern.

In this paper we have discussed the constraints imposed on neutrino masses and mixings when we demand the consistency with the LSND and the other terrestrial neutrino experiments and the flavor mixing solutions to the solar neutrino problem. Independent of the choice of the three solar neutrino solutions, the small- and the large-angle MSW, and the vacuum oscillation solutions, $s_{13}^{2}$ is constrained to be small, $s_{13}^{2} \lesssim \delta \sim 10^{-2}$, for different reasons in the MSW and in the vacuum solutions, respectively. The angle $s_{23}^{2}$ is subject to the constraint (15) which says that it is either small, $\lesssim 10^{-2}$ or large, $\sim 1$. On the other hand, the value of $s_{12}^{2}$ depends upon the solar neutrino solutions; $s_{12}^{2} \simeq 1.8 \times 10^{-3}, 0.18,0.5$ for the small-angle MSW, the large-angle MSW, and the vacuum oscillation solutions, respectively.

The physical interpretation of the solution is clearest in the small-angle MSW solution. The $\nu_{1}$ state is approximately identical with " $\nu_{e}$ ", and $\nu_{2}$ and $\nu_{3}$ are in general mixtures of " $\nu_{\mu}$ " and " $\nu_{\tau}$ ". Therefore, the type-a contains an inverted mass hierarchy and the type-b implies a normal mass pattern. Likewise one can obtain analogous physical interpretations in other solutions but the $\nu_{1}$ states is less pure with larger mixing angles.

Finally, a few remarks are in order: 
(1) The present analysis is less powerful in constraining the absolute values of masses than the relative masses and the mixing angles, a general feature noticed in [16]. The constraints obtained in this paper do allow, for Dirac neutrinos, the type-b solution with e.g., $m_{1}=6$ $\mathrm{eV}, m_{2}=6 \mathrm{eV}$, and $m_{3}=6.5 \mathrm{eV}$, which is consistent with the direct mass measurement [25] and the cosmological constraints [26]. This extreme choice would provide the possibility that the light neutrinos could fill the entire part of the missing mass in the universe, but with possible troubles with galaxy formation [26,3].

(2) One must be careful in comparing the resulting constraints obtained in this paper with that of Ref. [16] not only because they stand on entirely different basis but also because the definition of the angles are different. This complication arises due to the fact that we are working with the different definitions of the mass eigenstates here and in Ref. [16]. We are planning to present a unified and more transparent description in the future [27].

I thank Hiroshi Nunokawa for discussions, Eligio Lisi and the referee of Ref. [16 for calling my attention to various terrestrial experiments which have not addressed in the earlier version of this paper. This work is supported in part by Grant-in-Aid for Sciectific Research of the Ministry of Education, Science and Culture, \#0560355.

Note Added: After submitting the earlier version of this paper there appeared two reports from the LSND group [28,29] with mutually conflicting conclusions. 


\section{REFERENCES}

[1] New York Times, Tuesday 31, January 1995.

[2] W. C. Louis, Nucl. Phys. B (Proc. Suppl.) 38 (1995) 229; D. O. Caldwell, XXXth Rencontres de Moriond, January 21-28, 1995, Villars, Switzerland.

[3] J. R. Primack, J. Holtzman, A. Klypin, and D. O. Caldwell, Phys. Rev. Lett. 74 (1995) 2160; G. M. Fuller, J. R. Primack, and Y. -Z. Qian, preprint astro-ph/9502081; G. Raffelt and J. Silk, preprint hep-th/9502306; D. O. Caldwell and R. N. Mohapatra, preprint UCSB-HEP-95-1, hep-ph/9503316.

[4] J. N. Bahcall, Neutrino Astrophysics (Cambridge U. P., Cambridge, 1989).

[5] J. N. Bahcall, Phys. Lett. B338 (1994) 276; S. Parke, Phys. Rev. Lett. 74 (1995) 839; X. Shi, D. N. Schramm, and D. S. P. Dearborn, Phys. Rev. D50 (1994) 2414.

[6] B. T. Cleveland et al., Nucl. Phys. B (Proc. Suppl.) 38 (1995) 47.

[7] K. S. Hirata et al., Phys. Rev. D44 (1991) 2241; Y. Suzuki, Nucl. Phys. B (Proc. Suppl.) 38 (1995) 54.

[8] A. I. Abazov et al., Phys. Rev. Lett. 67 (1991) 3332; J. N. Abdurashitov et al., Nucl. Phys. B (Proc. Suppl.) 38 (1995) 60.

[9] P. Anselmann et al., Phys. Lett. B285 (1992) 376; ibid 327, (1994) 377.

[10] K. S. Hirata et al., Phys. Lett. B205 (1988) 416; ibid B280 (1992) 146.

[11] D. Casper et al., Phys. Rev. Lett. 66 (1991) 2561; R. Becker-Szendy et al., Phys. Rev. D46 (1992) 3720.

[12] M. C. Goodman, Nucl. Phys. B (Proc. Suppl.) 38 (1995) 337.

[13] Y. Fukuda et al., Phys. Lett. B335 (1994) 237.

[14] B. Achkar et al., Nucl. Phys. B434 (1995) 503. 
[15] F. Dydak et al., Phys. Lett. 134B (1984) 281.

[16] H. Minakata, preprint TMUP-HEL-9502, hep-ph/9503417.

[17] S. P. Mikheyev and A. Smirnov, Nuovo Cim. 9C (1986) 17; L. Wolfenstein, Phys. Rev. D17 (1978) 2369.

[18] N. Hata and P. Langacker, Phys. Rev. D50 (1994) 632; See also, G. L. Fogli, E. Lisi, and D. Montanino, Phys. Rev. D49 (1994) 3626.

[19] For recent analyses, V. Barger, R. J. N. Phillips and K. Whisnant, Phys. Rev. D43 (1991) 1110; A. Acker, S. Pakvasa, and J. Pantaleone, Phys. Rev. D43 (1991) 1754; P. I. Krastev and S. T. Petcov, Phys. Rev. Lett. 72 (1994) 1960.

[20] Review of Particle Properties, Phys. Rev. D50 (1994) 1315.

[21] T. K. Kuo and J. Pantaleone, Phys. Rev. D35 (1987) 3432.

[22] N. Ushida et al, Phys. Rev. Lett. 57 (1986) 2897.

[23] P. I. Krastev and S. T. Petcov, in Ref. [19].

[24] M. K. Moe, Nucl. Phys. B (Proc. Suppl.) 38 (1995) 36.

[25] E. W. Otten, Nucl. Phys. B (Proc. Suppl.) 38 (1995) 26.

[26] E. W. Kolb and M. S. Turner, The Early Universe (Addison-Wesley Publishing Co., California, 1990).

[27] H. Minakata, in preparation.

[28] C. Athanassopoulos et al., preprint NUCLEX/9504002.

[29] J. E. Hill, preprint hep-ex/9504009. 